\title{
Prosthetic heart valves in pregnancy: a systematic review and meta-analysis protocol
}

\author{
Claire M Lawley ${ }^{1,2^{*}}$, Samantha J Lain ${ }^{1}$, Charles S Algert ${ }^{1}$, Jane B Ford ${ }^{1}$, Gemma A Figtree ${ }^{2}$ and Christine L Roberts ${ }^{1}$
}

\begin{abstract}
Background: Advances in surgical technique, prosthetic heart valve design, and anticoagulation have contributed to an overall improvement in morbidity and mortality in women with heart valve prostheses as well as increased feasibility of pregnancy. Previous work investigating the pregnancies of women with prosthetic valves has been directed largely toward understanding the influence of anticoagulation regimen. There has been little investigation on maternal and infant outcomes. The objective of this systematic review will be to assess the outcomes of pregnancy in women with heart valve prostheses in contemporary populations.

Methods/Design: A systematic search of Medline, Embase, Cumulative Index to Nursing and Allied Health Literature (CINAHL), and the Cochrane Library will be undertaken. Article titles and abstracts will be evaluated by two reviewers for potential relevance. Studies that include pregnancies occurring from 1995 onwards and where there are six or more pregnancies in women with heart valve prostheses included in the study population will be reviewed for potential inclusion. Primary outcomes of interest will be mortality (maternal and perinatal). Secondary outcomes will include other pregnancy outcomes. No language restrictions will be applied. Methodological quality and heterogeneity of studies will be assessed. Data extraction from identified articles will be undertaken by two independent reviewers using a uniform template. Meta-analyses will be performed to ascertain risk of adverse events and, where numbers are sufficient, by type of prosthesis and location as well as other subgroup analyses.

Discussion: Estimates of the risk of adverse events in recent pregnancies of women with heart valve prosthesis will provide better information for counselling and decision making. Given the improvements in prognosis of heart valve prosthesis recipients and the paucity of definitive data regarding optimal pregnancy management for these women, review of this topic is pertinent.
\end{abstract}

Review registration: This protocol has been registered with the international prospective register of systematic reviews (PROSPERO) as number CRD42013006187, accessible online at http://www.crd.york.ac.uk/PROSPERO/ display_record.asp?ID=CRD42013006187\#.Utk7qNJ9Lf8.

\section{Background}

The etiology of valvular heart disease and management of congenital heart disease in young women continues to change. Advances in surgical technique, prosthetic heart valve design, and anticoagulation have contributed to an overall improvement in morbidity and mortality [1-4]. The number of women with heart valve prostheses counselled explicitly against pregnancy is decreasing with improvement in understanding of what conditions

\footnotetext{
* Correspondence: claw2317@uni.sydney.edu.au

${ }^{1}$ Clinical Population Perinatal Health Research Group, The Kolling Institute,

University of Sydney at Royal North Shore Hospital, Herbert Street,

St Leonards, New South Wales 2065, Australia

${ }^{2}$ Department of Cardiology, Royal North Shore Hospital, Herbert Street,

St Leonards, New South Wales 2065, Australia
}

and cardiac parameters constitute high risk of adverse events during pregnancy $[5,6]$. The focus is shifting to provision of informed decision making around the risk pregnancy might place on women and their babies. With these changes in mind, understanding the outcomes of pregnancies in women who have heart valve prostheses in the contemporary setting is of increasing relevance.

During normal pregnancy, there is an increase in hemodynamic load, which continues to rise during labor. This is as a result of increases in stroke volume and heart rate, increasing cardiac output by an estimated 30\% to $40 \%$, combined with a decrease in total peripheral resistance, leading to a decrease in blood pressure [7-9]. Pregnancy is a pro-coagulant state due to an elevation in

\section{Biomed Central}

(c) 2014 Lawley et al.; licensee BioMed Central Ltd. This is an open access article distributed under the terms of the Creative Commons Attribution License (http://creativecommons.org/licenses/by/2.0), which permits unrestricted use, distribution, and reproduction in any medium, provided the original work is properly cited. 
circulating pro-coagulant factors and maternal hormones, leading to decreases in prothrombin time, activated partial thromboplastin time, thrombin time, and international normalized ratio (INR) $[10,11]$. Tolerance of these hemodynamic and coagulatory changes in women with preexisting heart disease, including those with bioprosthetic and mechanical heart valve prostheses, is known to vary with underlying cardiac function and etiology of cardiac disease [6].

Previous work surrounding heart valve prostheses in pregnancy has been largely directed toward understanding the influence of anticoagulation type in the setting of mechanical heart valve prostheses [12-16]. A systematic review published in 2000, including 976 women who had 1,234 pregnancies, focused on maternal and fetal complications associated with various anticoagulation regimens [17]. The review included studies with pregnancies occurring from 1966 to 1997. As such, a large number of study participants (433/976) had older-generation and more thrombogenic cage-and-ball heart valve prostheses. Pooled analysis from this work demonstrated higher rates of fetal malformation in those women treated with oral anticoagulation in the first trimester (6.4\%, confidence interval (CI) 95\% 4.6\%-8.9\%, of pregnancies) as compared with where heparin was used in the first trimester $(3.4 \%$, CI 95\% 1.4\%-7.7\%, of pregnancies) [17]. A higher risk of thromboembolic complications was noted with heparin use. Another more recent review (2011) using pooled data from 959 pregnancies receiving oral anticoagulation throughout pregnancy and 285 pregnancies receiving unfractionated heparin in the first trimester found an incidence of maternal thromboembolic complications in 3.9\% and $9.5 \%$ of pregnancies in each group, respectively [15].

In the setting of mechanical heart valve prostheses in pregnancy, there is consensus in current international guidelines that one of three anticoagulation regimens may be used following assessment of maternal risk factors and preference: oral anticoagulation throughout pregnancy, oral anticoagulation with replacement by low-molecular-weight heparin or unfractionated heparin during weeks 6 to 12, or low-molecular-weight heparin or unfractionated heparin throughout pregnancy. Each of these regimens requires counseling around risk and judicious monitoring throughout, including INR and antifactor Xa levels where applicable $[5,18,19]$. The Royal College of Obstetricians recommends that women be offered a choice of one of these three regimens with education around the risks and benefits of each [20].

Bioprosthetic heart valves avoid the need for anticoagulation during pregnancy $[19,21]$. However, their use in younger patients has previously been limited due to the increased need for re-replacement compared with their mechanical counterparts [22,23]. This has been demonstrated in young women specifically, with $82 \%$ (CI 95\%
62\%-92\%) of women with bioprosthetic valve prostheses requiring replacement at 10 years as opposed to only $29 \%$ (CI 95\% 17\%-39\%) of women with mechanical valves [22]. Despite initial suggestions, recent work has not shown an increase in the rate of bioprosthetic valve deterioration in women undertaking pregnancies as opposed to women who do not $[24,25]$. With decreasing mortality and morbidity associated with valve re-replacement, international guidelines suggest that bioprosthetic valves be considered when heart valve replacement is required in women who may wish to become pregnant $[5,26]$.

Little work has been done exploring the population of contemporary heart valve recipients undertaking pregnancy. Specifically, other than anticoagulant type [12-17], there has been little investigation around rates and risk factors for maternal and infant adverse events. Given the changes in heart valves used, improvement in prognosis of contemporary heart valve prosthesis recipients, and the paucity of data in regarding the outcomes of pregnancies in these women, review of the studies in this area is warranted.

\section{Objectives}

Primary: To assess the risks of adverse outcomes of pregnancy among women with a prosthetic heart valve (s) in the contemporary setting.

Secondary: To assess the risks and relative risks of adverse outcomes of pregnancy in women with a prosthetic heart valve(s) by prosthesis type or location or both.

\section{Methods/Design}

Study registration

This protocol has been registered with the international prospective register of systematic reviews (PROSPERO) as number CRD42013006187.

The systematic review protocol has been conducted and reported by using the Preferred Reporting Items for Systematic Reviews and Meta-Analyses (PRISMA) [27] where applicable and the Meta-analysis of Observational Studies in Epidemiology (MOOSE) reporting guidelines [28].

\section{Outcomes of interest}

Primary outcomes:

1) Maternal mortality

2) Any pregnancy loss

a. Any loss of pregnancy, including miscarriage/stillbirth/termination of pregnancy [29] (or as defined by study)

3) Perinatal mortality [29]

a. Stillbirth: Fetal death in utero at least 22 weeks of gestation [29] (or as defined by the study) 
b. Neonatal mortality: Death in the first 28 days of extra-uterine life [29]

c. Perinatal mortality: Stillbirth or neonatal mortality

Secondary outcomes:

1) Adverse maternal outcomes

a. Any thromboembolic events, including

i. Stroke/Transient ischemic event

ii. Valve thrombosis

iii. Other

b Any obstetric hemorrhage, including

i. Antenatal hemorrhage

ii. Postpartum hemorrhage

c. Cardiovascular compromise (as defined by study)

d. Valve deterioration (bioprosthetic valves only, as defined by study)

e. New arrhythmia

f. Infective endocarditis

g. Myocardial infarction

h. Pregnancy hypertension, including gestational hypertension, pre-eclampsia, and eclampsia

2) Labor and delivery outcomes (including those

subject to clinical decision making)

a. Mode of delivery

3) Adverse birth outcomes

a. Preterm birth

Delivery before 37 weeks of gestation

b. Small for gestational age

Less than tenth birth weight percentile for sex and gestational age

c. Low birth weight

Birth weight less than 2,500 grams

d. Infant admission to neonatal intensive care unit

e. Congenital malformation

Search strategy for identification of studies and methods of review

A systematic search of Medline, Embase, Cumulative Index to Nursing and Allied Health Literature (CINAHL), and the Cochrane Library will be undertaken to identify relevant studies published between 1995 and May 2013. Search terms will include "pregnancy" AND ("heart valves" OR "heart valve replacement" OR "heart valve prosthesis" OR "heart valve prosthesis implantation"). The "explode" function will be used in each case. Searches will be limited to studies of humans and peer-reviewed articles. Language restrictions will not be applied, and every effort will be made to obtain translations; articles unable to be translated will be reported. Duplicates will be removed.

\section{Eligibility criteria for consideration of inclusion}

Study types:

Studies that report outcomes of women with prosthetic valves undertaking pregnancy:

- Randomized controlled trials

- Clinical trials

- Cohort studies

- Cross-sectional studies

- Unselected case series

Studies that compare outcomes for women with prosthetic valves by valve location or type:

- Randomized controlled trials

- Clinical trials

- Cohort studies

- Case-control studies

Control or comparison groups are not necessary to the primary objective of estimating risk among women with valve prosthesis. It is anticipated that most of the studies identified for consideration will be case series.

Populations: Populations of pregnant women that include women with prosthetic heart valves.

Comparators:

Where studies differentiate between mechanical and biological valve prosthesis, or between valve location (that is, mitral, aortic, pulmonary, and tricuspid), applicable to the secondary objective; relative risks by prosthesis type or location or both, relative risks of adverse events will be calculated by using:

- Biological prosthesis as the denominator for calculating relative risk compared with mechanical

- Presence of mitral valve prosthesis as the denominator for calculating relative risk compared with other valve locations

Study criteria:

- Include pregnancies occurring from 1995 onwards only.

- Contain at least six pregnancies in women with heart valve prostheses in the study population. This was chosen as it has been used in a systematic review exploring anticoagulation regimens during pregnancy in women with heart valve prostheses [17].

- Study population should have fewer than $5 \%$ of women with a Starr-Edwards (cage-and-ball) heart valve prosthesis. This was a pragmatic decision. Owing to high thrombogenic complication rates, cage-and-ball prostheses are no longer implanted 
$[30,31]$ and therefore are not relevant when evaluating in the contemporary setting. Consequently, where the study population consists of not more than 20 women, if one (5\%) or more participants has a cage-and-ball valve, the study will be excluded.

- Where a case series is presented, participants have not been selected due to the occurrence of an adverse event (for example, valve thrombosis during pregnancy).

- Not a conference abstract and unpublished study.

Exposure of interest: Pregnancy in women with a heart valve prosthesis.

\section{Screening of studies}

Article titles and abstracts will be evaluated by two reviewers for potential relevance. Where there is disagreement at this stage, the article will remain included until the full text is reviewed prior to a decision. In this process, articles relating to other aspects of valvuar heart disease and pregnancy but not specific to maternal vavular prostheses will be excluded (for example, articles related to fetal cardiac disease or basic science). Articles identified through reference lists of included studies and relevant systematic reviews will be considered for inclusion on the basis of their title.

At least two independent reviewers will assess all articles identified in the screening process for potential inclusion, including assessment of methodological quality as outlined below. Where information pertinent to inclusion criteria is not contained within the article text, the effort will be made to contact the listed corresponding author. Where no reply is received, the article will be excluded. Consensus between the two authors undertaking review of the study will need to be reached before the article is included. In the event that a consensus is not reached, a third reviewer will be involved as an arbitrator. A flow chart of the study selection procedure will be prepared and a log of rejected studies maintained.

\section{Data extraction}

Data extraction from identified articles will be undertaken by two independent reviewers using a uniform template. Discrepancies will be resolved by discussion and, where applicable, arbitration by a third reviewer.

The following information will be extracted:

Study characteristics: authors, year of publication, study design, location, and time period of included pregnancies

Population characteristics: number of participants, number of pregnancies, maternal age, and parity
Heart valve characteristics: number of mechanical valves, number of bioprosthetic valves, implanted valve type, implanted valve location, and anticoagulation regimen Adverse outcomes: frequency of adverse outcomes as outlined above.

\section{Assessment of methodological quality}

It is thought likely that the only randomized studies eligible for inclusion will be randomized control trials assessing different valve types. The risk of bias in randomized studies will be assessed by using the Cochrane Collaboration's tool for assessing risk of bias [32]. This tool provides a model to evaluate the risk of bias across a number of domains: how a study selects participants, measures performance, blinds participants and investigators, explores attrition, and reports findings. Each domain for each study will be allocated a ranking of "low", "unclear", or "high" risk of bias, in accordance with the Cochrane Collaboration's approach, by two separate reviewers. Where there is a discrepancy between the two reviewers, a third reviewer will be used as an arbitrator.

Included non-randomized studies may or may not have a comparison group. To assess the risk of bias within included these studies, the methodological quality of potential studies will be assessed by using the Newcastle-Ottawa scale (NOS) for assessing the quality of non-randomized studies in meta-analyses [33]. The NOS for case-control and cohort studies will be adapted (Table 1) to meet the specific needs of this systematic review. The cohort scale will be modified for use in case series [33]. Using the NOS, studies will be awarded a maximum of nine points on items related to the selection of the study groups, the comparability of the groups, and the ascertainment of outcome of interest. Using this modified score, case series will be eligible for a maximum of six points. This will be undertaken by two separate reviewers. Where there is disagreement, a third reviewer will be used as an arbitrator.

\section{Data analysis and presentation}

A table with descriptive information for each study will be produced Additional file 1: Table S1. From extracted data, the risk of outcomes for the primary objective will be calculated by dividing the total number of outcome occurrences by the total number of pregnancies or births to women with a heart valve prosthesis. The risk of maternal mortality and any pregnancy loss will be expressed as the proportion of the total number of pregnancies (including miscarriages, terminations, stillbirths, and live births). The risk of perinatal death and secondary adverse birth outcomes (as opposed to pregnancy outcomes) will be expressed as a proportion of the pregnancies beyond 22 weeks of gestation or 500 grams or resulting in a live 
Table 1 Adapted Newcastle-Ottawa scale [33] for "Prosthetic heart valves in pregnancy: a systematic review and meta-analysis protocol"

\begin{tabular}{|c|c|}
\hline Criteria & $\begin{array}{c}\text { Star allocated } \\
\text { (maximum } 9 \text { stars) }^{\mathrm{a}}\end{array}$ \\
\hline \multicolumn{2}{|l|}{ Selection } \\
\hline \multicolumn{2}{|l|}{ 1) Representativeness of the exposed cohort } \\
\hline $\begin{array}{l}\text { a) Population truly representative of pregnant } \\
\text { women with prosthetic heart valves }\end{array}$ & $\star$ \\
\hline $\begin{array}{l}\text { b) Somewhat representative of the population } \\
\text { of pregnant women with prosthetic heart } \\
\text { valves }\end{array}$ & $\star$ \\
\hline $\begin{array}{l}\text { c) Selected group of users (for example, } \\
\text { referral hospital patients) }\end{array}$ & - \\
\hline $\begin{array}{l}\text { d) No description of the derivation of } \\
\text { the cohort }\end{array}$ & - \\
\hline \multicolumn{2}{|l|}{ 2) Selection of the non-exposed cohort ${ }^{b}$} \\
\hline $\begin{array}{l}\text { a) Drawn from the same community as } \\
\text { the exposed cohort }\end{array}$ & $\star$ \\
\hline b) Drawn from a different source & - \\
\hline $\begin{array}{l}\text { c) No description of the derivation of the } \\
\text { non-exposed cohort }\end{array}$ & - \\
\hline d) Not applicable & - \\
\hline \multicolumn{2}{|l|}{ 3) Ascertainment of exposure } \\
\hline $\begin{array}{l}\text { a) Secure record (for example, } \\
\text { medical records) }\end{array}$ & $\star$ \\
\hline b) Structured interview & $\star$ \\
\hline c) Written self-report & - \\
\hline d) No description & - \\
\hline \multicolumn{2}{|c|}{$\begin{array}{l}\text { 4) Demonstration that outcome of interest was not present at start } \\
\text { of studyc }\end{array}$} \\
\hline a) Yes & $\star$ \\
\hline b) No & - \\
\hline c) Not applicable & - \\
\hline \multicolumn{2}{|l|}{ Comparability } \\
\hline \multicolumn{2}{|c|}{ Comparability of cohorts on the basis of the design or analysis } \\
\hline $\begin{array}{l}\text { a) Study controls for maternal age (select the } \\
\text { most important factor) }\end{array}$ & $\star$ \\
\hline $\begin{array}{l}\text { b) Study controls for any additional } \\
\text { factor (type of valve, valve location, } \\
\text { anticoagulation regimen) }\end{array}$ & $\star$ \\
\hline c) Not applicable & - \\
\hline \multicolumn{2}{|l|}{ Outcome } \\
\hline \multicolumn{2}{|l|}{ 1) Assessment of outcome } \\
\hline a) Independent blind assessment & $\star$ \\
\hline b) Record linkage & $\star$ \\
\hline c) Self-report & - \\
\hline d) No description & - \\
\hline \multicolumn{2}{|c|}{$\begin{array}{l}\text { 2) Was follow-up long enough for outcomes (as defined by study) } \\
\text { to occur? }\end{array}$} \\
\hline a) Yes & $\star$ \\
\hline b) No & - \\
\hline
\end{tabular}

Table 1 Adapted Newcastle-Ottawa scale [33] for "Prosthetic heart valves in pregnancy: a systematic review and meta-analysis protocol" (Continued)

\begin{tabular}{|c|c|}
\hline \multicolumn{2}{|l|}{ 3) Adequacy of follow up of cohorts } \\
\hline $\begin{array}{l}\text { a) Complete follow up (all subjects accounted } \\
\text { for and no missing data) }\end{array}$ & $\star$ \\
\hline $\begin{array}{l}\text { b) Subjects lost to follow-up unlikely to } \\
\text { introduce bias - small number lost - } \geq 80 \%\end{array}$ & $\star$ \\
\hline c) Follow-up rate $<80 \%$ & - \\
\hline d) No statement & - \\
\hline \multicolumn{2}{|c|}{$\begin{array}{l}\text { aA study can be awarded a maximum of one star for each numbered item } \\
\text { within the selection and outcome categories. A maximum of two stars can be } \\
\text { given for comparability. "For example, women without a heart valve prosthesis } \\
\text { undertaking pregnancy. Likely to be "not applicable" for some study types, } \\
\text { including case series. "Suggested primary outcomes: maternal mortality, any } \\
\text { pregnancy loss, and perinatal mortality. }\end{array}$} \\
\hline
\end{tabular}

birth [29]. It is anticipated that these denominators may not always be clearly articulated, potentially constraining the process to what is reported in each study.

Subgroup analysis of primary and secondary outcome relative risks by valve location and valve type will be undertaken if reported by at least two studies, each with at least six pregnancies in the subgroup. Comprehensive Meta-Analysis (version 2.0) software (Biostat, Englewood, NJ, USA) be used for the data analysis. This software enables pooling of risks as well as of relative risks, making it suitable for our primary objective, especially as it is anticipated that the majority of studies will be case series. Pooled risks will be calculated by using a random effects model, with variance calculated by using a logit conversion. Graphic summaries of individual study estimates and overall estimates will be produced. Statistical uncertainty will be assessed by using 95\% confidence intervals around risk estimates.

Where applicable, heterogeneity of effect for studies within a meta-analysis will be assessed with the $\mathrm{I}^{2}$ statistic. Study heterogeneity will be explored by categorization of the study design, the year of publication, the time period within which pregnancies occur, and population characteristics (ethnicity, age range, etiology of underlying disease, type and location of heart valve prosthesis, and anticoagulant regimen). It is expected that study characteristics will vary and that random effects models will be appropriate for estimating overall event risks.

In general, the strength of evidence will be assessed with respect to the study designs, the methodological quality of the individual studies, the consistency of the results across studies, and, for studies with a comparison or control group, the strength of associations. More specifically, given the likelihood that most studies will be uncontrolled case series, the strength of evidence will be assessed primarily by the width of the confidence interval around pooled outcome rates. Consistency of effect 
will also be important both as demonstrated visually in the plots and as quantified by the $\mathrm{I}^{2}$ statistic.

\section{Discussion}

Improved care for chronic diseases and delayed age of childbearing has contributed to an increase in the number of pregnant women with concurrent medical conditions, including valvular heart disease. The proposed systematic review is of importance in the context of global pressure to improve maternal and infant health, including the evaluation of pregnancies in subgroups of women with co-morbidities. In Australia, this is seen through the prioritization of research work encompassing "Healthy start to life for all Australians" [20].

Meta-analyses of observational studies present challenges because of inherent biases within different study designs [34]. Nevertheless, they help understanding and quantify variation in results between studies [28]. In the context of predominantly observational studies, it is thus essential that a rigorous protocol be designed to address the outcome of pregnancies in women with heart valve prostheses.

Through exploration of the outcomes of pregnancies in women with heart valve prosthesis in the contemporary setting (1995 onwards), this systematic review will provide estimates of the risk of adverse events in these pregnancies. It is hoped that this information will improve the understanding of risk factors for poor maternal, pregnancy, and infant outcomes, thereby providing information for clinical decision making and patient counselling. It is timely that this work is undertaken given the developments in heart valve prosthesis technology, overall improvement in prognosis of young women with a heart valve prosthesis, and increases in the number of women with congenital heart disease reaching reproductive age.

\section{Additional file}

Additional file 1: Table S1. Sample of table to record descriptive information extracted from each included study.

\section{Abbreviations}

Cl: confidence interval; INR: international normalized ratio; NOS: NewcastleOttawa scale.

\section{Competing interests}

The authors declare that they have no competing interests.

\section{Authors' contributions}

CR and GF helped to conceive of the study and were involved in the design of the above review protocol and in drafting and revising the manuscript. $C L, S L, C A$, and JF were involved in the design of the above review protocol and in drafting and revising the manuscript. All authors read and approved the final manuscript.

\section{Acknowledgments}

This work was supported by an Australian National Health and Medical Research Council (NHMRC) Centre for Research Excellence Grant (\#1001066). CR is supported by an NHMRC Senior Research Fellowship (\#APP1021025) and JF is supported by an Australian Research Council Future Fellowship (\#FT120100069). The funding agencies listed had no role in protocol design, decision to publish, or preparation of the manuscript.

Received: 2 September 2013 Accepted: 6 January 2014

Published: 21 January 2014

\section{References}

1. Fernandes SM, Pearson DD, Rzeszut A, Mitchell SJ, Landzberg MJ, Martin GR, American College of Cardiology, Adult Congenital Heart Disease Working Group; Adult Congenital Cardiac Care Associate Research Network: Adult congenital heart disease incidence and consultation: a survey of general adult cardiologists. J Am Coll Cardiol 2013, 61:1303-1304.

2. Marelli AJ, Mackie AS, lonescu-lttu R, Rahme E, Pilote L: Congenital heart disease in the general population: changing prevalence and age distribution. Circulation 2007, 115:163-172.

3. Khairy P, lonescu-lttu R, Mackie AS, Abrahamowicz M, Pilote L, Marelli AJ: Changing mortality in congenital heart disease. J Am Coll Cardiol 2010, 56:1149-1157.

4. Ruel M, Kulik A, Lam BK, Rubens FD, Hendry PJ, Masters RG, Bédard P, Mesana TG: Long-term outcomes of valve replacement with modern prostheses in young adults. Eur J Cardiothorac Surg 2005, 27:425-433. Discussion 433.

5. Bonow RO, Carabello BA, Chatterjee $\mathrm{K}$, de Leon AC Jr, Faxon DP, Freed MD, Gaasch WH, Lytle BW, Nishimura RA, O'Gara PT, O'Rourke RA, Otto CM, Shah PM, Shanewise JS, American College of Cardiology/American Heart Association Task Force on Practice Guidelines: 2008 Focused update incorporated into the ACC/AHA 2006 guidelines for the management of patients with valvular heart disease: a report of the American college of cardiology/American heart association task force on practice guidelines (writing committee to revise the 1998 guidelines for the management of patients with valvular heart disease). endorsed by the society of cardiovascular anesthesiologists, society for cardiovascular angiography and interventions, and society of thoracic surgeons. J Am Coll Cardiol 2008, 52:e1-e142.

6. Siu SC, Sermer M, Colman JM, Alvarez AN, Mercier LA, Morton BC, Kells CM, Bergin ML, Kiess MC, Marcotte F, Taylor DA, Gordon EP, Spears JC, Tam JW, Amankwah KS, Smallhorn JF, Farine D, Sorensen S, Cardiac Disease in Pregnancy (CARPREG) Investigators: Prospective multicenter study of pregnancy outcomes in women with heart disease. Circulation 2001, 104:515-521.

7. Gilson GJ, Samaan S, Crawford MH, Qualls CR, Curet LB: Changes in hemodynamics, ventricular remodeling, and ventricular contractility during normal pregnancy: a longitudinal study. Obstet Gynecol 1997 89:957-962

8. Abbas AE, Lester SJ, Connolly H: Pregnancy and the cardiovascular system. Int J Cardiol 2005, 98:179-189.

9. Poppas A, Shroff SG, Korcarz CE, Hibbard JU, Berger DS, Lindheimer MD, Lang RM: Serial assessment of the cardiovascular system in normal pregnancy: role of arterial compliance and pulsatile arterial load. Circulation 1997, 95:2407-2415.

10. Hui C, Lili M, Libin C, Rui Z, Fang G, Ling G, Jianping Z: Changes in coagulation and hemodynamics during pregnancy: a prospective longitudinal study of 58 cases. Arch Gynecol Obstet 2012, 285:1231-1236.

11. Bremme KA: Haemostatic changes in pregnancy. Best Pract Res Clin Haematol 2003, 16:153-168.

12. Basude S, Hein C, Curtis SL, Clark A, Trinder J: Low-molecular-weight heparin or warfarin for anticoagulation in pregnant women with mechanical heart valves: what are the risks? a retrospective observational study. BJOG 2012, 119:1008-1013. Discussion 1012-1013.

13. Yinon Y, Siu SC, Warshafsky C, Maxwell C, McLeod A, Colman JM, Sermer M, Silversides CK: Use of low molecular weight heparin in pregnant women with mechanical heart valves. Am J Cardiol 2009, 104:1259-1263.

14. Quinn J, Von Klemperer K, Brooks R, Peebles D, Walker F, Cohen H: Use of high intensity adjusted dose low molecular weight heparin in women with mechanical heart valves during pregnancy: a single-center experience. Haematologica 2009, 94:1608-1612. 
15. McLintock C: Anticoagulant therapy in pregnant women with mechanical prosthetic heart valves: no easy option. Thromb Res 2011 127(suppl):S56-S60.

16. Castellano JM, Narayan RL, Vaishnava P, Fuster V: Anticoagulation during pregnancy in patients with a prosthetic heart valve. [Review]. Nat Rev Cardiol 2012, 9:415-424.

17. Chan WS, Anand S, Ginsberg JS: Anticoagulation of pregnant women with mechanical heart valves: a systematic review of the literature. [Review] [47 refs]. Arch Intern Med 2000, 160:191-196.

18. Bates SM, Greer IA, Pabinger I, Sofaer S, Hirsh J, American College of Chest Physicians: Venous thromboembolism, thrombophilia, antithrombotic therapy, and pregnancy: American college of chest physicians evidence-based clinical practice guidelines (8th edition). Chest 2008, 133:844S-886S.

19. European Society of Gynecology (ESG); Association for European Paediatric Cardiology (AEPC); German Society for Gender Medicine (DGesGM), Regitz-Zagrosek V, Blomstrom Lundqvist C, Borghi C, Cifkova R, Ferreira R, Foidart JM, Gibbs JS, Gohlke-Baerwolf C, Gorenek B, lung B, Kirby M, Maas AH, Morais J, Nihoyannopoulos P, Pieper PG, Presbitero P Roos-Hesselink JW, Schaufelberger M, Seeland U, Torracca L, ESC Committee for Practice Guidelines: ESC guidelines on the management of cardiovascular diseases during pregnancy. Eur Heart J 2011, 32:3147-3197.

20. National Health and Medical Research Council: A healthy start to life for all Australians. [http://www.nhmrc.gov.au/grants/outcomes-funding-rounds/ historical-information-capacity-building-grants/-healthy-start-life-a]

21. Joint Task Force on the Management of Valvular Heart Disease of the European Society of Cardiology (ESC); European Association for CardioThoracic Surgery (EACTS), Vahanian A, Alfieri O, Andreotti F, Antunes MJ, Barón-Esquivias G, Baumgartner H, Borger MA, Carrel TP, De Bonis M, Evangelista A, Falk V, lung B, Lancellotti P, Pierard L, Price S, Schäfers HJ, Schuler G, Stepinska J, Swedberg K, Takkenberg J, Von Oppell UO, Windecker S, Zamorano JL, Zembala M: Guidelines on the management of valvular heart disease (version 2012). Eur Heart J 2012, 33:2451-2496.

22. North RA, Sadler L, Stewart AW, McCowan LM, Kerr AR, White HD: Long-term survival and valve-related complications in young women with cardiac valve replacements. Circulation 1999, 99:2669-2676.

23. Hammermeister K, Sethi GK, Henderson WG, Grover FL, Oprian C, Rahimtoola $\mathrm{SH}$ : Outcomes 15 years after valve replacement with a mechanical versus a bioprosthetic valve: final report of the veterans affairs randomized trial. J Am Coll Cardiol 2000, 36:1152-1158.

24. El SF, Hassan W, Latroche B, Helaly S, Hegazy H, Shahid M, Mohamed G, Al-Halees Z: Pregnancy has no effect on the rate of structural deterioration of bioprosthetic valves: long-term 18-year follow up results. J Heart Valve Dis 2005, 14:481-485.

25. Avila WS, Rossi EG, Grinberg M, Ramires JA: Influence of pregnancy after bioprosthetic valve replacement in young women: a prospective five-year study. J Heart Valve Dis 2002, 11:864-869.

26. Royal College of Obstetricians and Gynaecologists: Heart disease and pregnancy study group statement: consensus views arising from the 51st study group: heart disease and pregnancy. [http://www.rcog.org.uk/ womens-health/clinical-guidance/heart-disease-and-pregnancy-study-groupstatement]

27. Moher D, Liberati A, Tetzlaff J, Altman DG, PRISMA Group: Preferred reporting items for systematic reviews and meta-analyses: the PRISMA statement. BMJ 2009, 339:b2535.

28. Stroup DF, Berlin JA, Morton SC, Olkin I, Williamson GD, Rennie D, Moher D, Becker BJ, Sipe TA, Thacker SB: Meta-analysis of observational studies in epidemiology: a proposal for reporting: meta-analysis of observational studies in epidemiology (MOOSE) group. JAMA 2000, 283:2008-2012.

29. World Health Organization: Neonatal and Perinatal Mortality: Country Regional and Global Estimates. Geneva, Switzerland: World Health Organization; 2006.

30. Macmanus Q, Grunkemeier G, Thomas D, Lambert LE, Starr A: The Starr-Edwards model 6000 valve: a fifteen-year follow-up of the first successful mitral prosthesis. Circulation 1977, 56:623-625.

31. Pibarot P, Dumesnil JG: Prosthetic heart valves: selection of the optimal prosthesis and long-term management. Circulation 2009, 119:1034-1048.

32. Higgins JP, Altman DG, Gøtzsche PC, Jüni P, Moher D, Oxman AD, Savovic J, Schulz KF, Weeks L, Sterne JA, Cochrane Bias Methods Group; Cochrane
Statistical Methods Group: The Cochrane Collaboration's tool for assessing risk of bias in randomised trials. BMJ 2011, 343:d5928.

33. Wells GS, O'Connell D, Peterson J, Welch V, Losos M, Tugwell P: The Newcastle-Ottawa scale (NOS) for assessing the quality of nonrandomised studies in meta-analyses. Ottawa Hospital Research Institute. 2008 [http://www.ohri.ca/programs/clinical_epidemiology/oxford.asp]

34. Sanderson S, Tatt ID, Higgins JPT: Tools for assessing quality and susceptibility to bias in observational studies in epidemiology: a systematic review and annotated bibliography. Int J Epidemiol 2007, 36:666-676.

doi:10.1186/2046-4053-3-8

Cite this article as: Lawley et al:: Prosthetic heart valves in pregnancy: a systematic review and meta-analysis protocol. Systematic Reviews 2014 3:8.

\section{Submit your next manuscript to BioMed Central and take full advantage of:}

- Convenient online submission

- Thorough peer review

- No space constraints or color figure charges

- Immediate publication on acceptance

- Inclusion in PubMed, CAS, Scopus and Google Scholar

- Research which is freely available for redistribution 